This item was submitted to Loughborough's Research Repository by the author.

Items in Figshare are protected by copyright, with all rights reserved, unless otherwise indicated.

\title{
Dynamics of compartmental model recurrent neural networks
}

PLEASE CITE THE PUBLISHED VERSION

PUBLISHER

(C) American Physical Society

LICENCE

CC BY-NC-ND 4.0

REPOSITORY RECORD

Bressloff, P.C.. 2019. "Dynamics of Compartmental Model Recurrent Neural Networks". figshare. https://hdl.handle.net/2134/1714. 


\title{
Dynamics of compartmental model recurrent neural networks
}

\author{
Paul C. Bressloff \\ Department of Mathematical Sciences, Loughborough University of Technology, Loughborough, \\ Leicestershire LE11 3TU, United Kingdom
}

(Received 25 April 1994)

\begin{abstract}
We show how the dynamics of a recurrent network of compartmental model neurons can be formulated in terms of a set of coupled nonlinear Volterra integrodifferential equations in which the state of each neuron is represented by a single scalar variable. The associated convolution kernels are determined by the single neuron membrane potential response function, which can be calculated explicitly for arbitrary dendritic tree topologies. Our integral equation approach provides a compact and analytically tractable method for studying the effects of dendritic structure on network dynamics. We illustrate this by deriving conditions for the onset of oscillations in a compartmental model network that depend both on the interneuron connection weights and on the internal dendritic structure of the individual neurons. It is shown that it is possible for a symmetrically connected compartmental model network to oscillate.
\end{abstract}

PACS number(s): 87.10. $+\mathrm{e}, 02.30 . \mathrm{Ks}$

\section{INTRODUCTION}

Most neural network models consider a neuron to be a point processor that transforms a linear weighted sum of inputs into an output according to some nonlinear threshold function without delays. On the other hand, it is well known from neurophysiology that the passive membrane properties of a neuron's dendritic tree system leads to a diffusive spread of activity throughout the tree so that the neuron's response depends on previous input history (infinite delays) and the particular locations of the stimulated synapses within the dendritic tree. Thus dendritic structure influences the temporal processing of synaptic inputs. It is still poorly understood how this feature contributes to the information processing capabilities of real neurons.

A popular way of modeling the dendritic tree is to segment it into a set of compartments such that the spatial variation of the membrane potential and other physical quantities across each compartment is negligible [1]. Compartmental models can be described in terms of a set of coupled ordinary differential equations. Since these are particularly amenable to computer simulations, compartmental models form the basis of most numerical studies of the more realistic neuronal systems considered in computational neuroscience [2]. Recently, a method for calculating the response function of a compartmental model neuron has been developed, which yields simple analytical expressions that are applicable to trees of arbitrary topology and that have an explicit dependence on important neurophysiological parameters [3]. These expressions have been used to study the response of a neuron to time-varying inputs [4], as well as to analyze the effects of synaptic background activity [5] and shunting [6]. However, the mathematical analysis has tended to be at the single neuron level.

In this paper, we extend our previous work by analyzing the dynamics of a recurrent analogue neural network consisting of identical compartmental model neurons.
We show how the resulting set of ordinary differential equations can be reduced to a much smaller set of Volterra integrodifferential equations in which the state of each neuron is represented by a single scalar variable, namely, the somatic membrane potential; the dendritic potentials are treated as auxiliary variables. The kernels of the Volterra equations, which are of convolution type, are determined by the single neuron response function calculated in Ref. [3]. Hence, our integral equation formulation provides a compact and analytically tractable way of incorporating dendritic structure into recurrent neural network models. (It should be noted that Poggio and Torre [7] have also considered the representation of dendritic structure in terms of integral equations, however, their analysis is purely at the single neuron level and is concerned with the effects of shunting.)

Each neuron in the compartmental model network is effectively performing a temporal summation over all previous inputs to that neuron as expressed by the convolution integrals [4]. Thus, one can consider the network as having continuously distributed delays. One well known feature of delays it that they can lead to destabilization of an equilibrium and the simultaneous creation of a stable limit cycle via a supercritical Hopf bifurcation $[8,9]$. This was studied previously by Marcus and Westervelt [10] in the case of an analogue network with discrete delays. In this paper, we derive conditions for the onset of oscillations in a compartmental model network. These depend on both the interneuron connection weights and the internal dendritic structure of the individual neurons. We proceed by linearizing the Volterra integrodifferential equations about an equilibrium and constructing the associated characteristic equation. This involves the Laplace transform of the single neuron response function, which can be calculated explicitly for arbitrary dendritic trees using the results of Bressloff and Taylor [3]. The (local) stability of the equilibrium is then analyzed in terms of the roots of the characteristic equation, and a stability region in weight space established. However, as 
illustrated by Burton [11], linear stability analysis is itself insufficient to guarantee the existence of solutions with sustained oscillations; one must take into account the nonlinearities. We achieve this here by adapting a version of the Hopf bifurcation theorem due to Allwright [12], which again makes explicit use of the single neuron response function of Ref. [3]. In particular, we show how a compartmental model network with symmetric connection weights can oscillate.

The organization of the paper is as follows. The integral equation approach to compartmental model neurodynamics is presented in Sec. II. Some general results concerning the dynamics of Volterra integrodifferential equations are briefly described in Sec. III. These are then used in the linear stability analysis of a compartmental model network (Sec. IV). Finally, the Hopf bifurcation theorem for the onset of oscillations in a compartmental model network is developed in Sec. V.

\section{COMPARTMENTAL MODEL}

Consider a fully-connected network of identical compartmental model neurons labeled $i=1, \ldots, N$. Each neuron consists of a set of dendritic compartments coupled to a single somatic compartment where the process of action potential generation occurs. The complex topology of the dendrites is specified by a simply connected graph or tree $\Gamma$ ( $i$ independent). Each node of the tree labeled $\alpha \in \Gamma$ corresponds to a single dendritic compartment over which the spatial variation of the membrane potential and other physical quantities is negligible. The passive membrane properties of the $\alpha$ th compartment are represented in terms of an equivalent circuit consisting of a membrane leakage resistor $R_{\alpha}$ in parallel with a capacitor $C_{\alpha}$. Each compartment $\alpha$ is joined to its immediate neighbors $\beta$ in the tree by the junctional resistors $R_{\alpha \beta}$. The dendritic system is coupled to an additional somatic compartment by a single junctional resistor $r$ from dendritic compartment $\alpha_{0} \in \Gamma$. The soma has membrane leakage resistance $\hat{R}$ and capacitance $\hat{C}$ (the basic model of a neuron is shown in Fig. 1). Let $V_{i \alpha}$ be the membrane potential of compartment $\alpha$ belonging to the $i$ th neuron of the network, and let $U_{i}$ be the corresponding potential at the soma. Denote the synaptic weight of the connection from neuron $j$ to the $\alpha$ th compartment of neuron $i$ by $W_{i j}^{\alpha}$. The associated synaptic input is taken to be of the form $W_{i j}^{\alpha} f\left(U_{j}\right)$, where $f$ is the output function of each neuron. (Note that we are ignoring shunting terms, see Sec. VI). We shall take $f$ to be a smooth function of the somatic potential that saturates at $f= \pm 1$, that is

$$
f(U)=\tanh (\kappa U),
$$

where $\kappa$ is a gain parameter. The output $f(U)$ may be interpreted as the short term average firing rate of a neuron. Finally, $\hat{W}_{i j} f\left(U_{j}\right)$ is the synaptic input located at the soma.

Under the above assumptions, an application of Kirchoff's law yields a set of ordinary differential equations

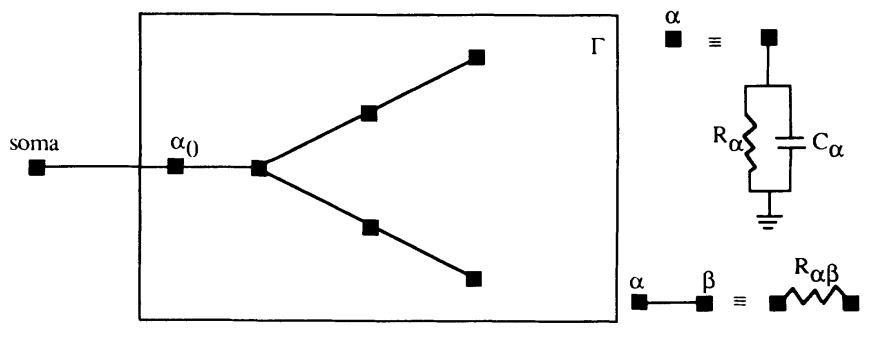

FIG. 1. Basic compartmental model of a neuron.

$$
\begin{aligned}
C_{\alpha} \frac{d V_{i \alpha}}{d t}= & -\frac{V_{i \alpha}(t)}{R_{\alpha}}+\sum_{\langle\beta \in \Gamma ; \alpha\rangle} \frac{V_{i \beta}(t)-V_{i \alpha}(t)}{R_{\alpha \beta}} \\
& +\frac{U_{i}(t)-V_{i \alpha_{0}}(t)}{r} \delta_{\alpha, \alpha_{0}} \\
& +\sum_{j} W_{i j}^{\alpha} f\left(U_{j}(t)\right)+I_{i \alpha}(t), \quad \alpha \in \Gamma \\
\hat{C} \frac{d U_{i}}{d t}=- & \frac{U_{i}(t)}{\hat{R}}+\frac{V_{i \alpha_{0}}(t)-U_{i}(t)}{r} \\
& +\sum_{j} \hat{W}_{i j} f\left(U_{j}(t)\right)+\hat{I}_{i}(t),
\end{aligned}
$$

where $I_{i \alpha}(t), \hat{I}_{i}(t)$ are external input currents. The summation over $\beta$ in Eq. (2.2) is restricted to nearest dendritic neighbors of $\alpha$ as indicated by the notation $\langle\beta ; \alpha\rangle$. One is now faced with the task of analyzing the set of $N(M+1)$ coupled nonlinear equations, where $M$ is the number of dendritic compartments per neuron. This can be quite formidable, especially when $M$ is large. For example, unlike the standard analog model [13], there is no simple Liapunov function that can be constructed in order to guarantee stability. However, one is really only concerned with the somatic potentials $U_{i}(t)$ since these determine the output of the network and are the source of the nonlinear terms. In other words, the dendritic potentials $V_{i \alpha}(t)$ can be viewed as auxiliary variables. Therefore, we shall proceed by eliminating the auxiliary variables $V_{i \alpha}(t)$, which appear linearly in Eqs. (2.2) and (2.3), to obtain an integrodifferential equation for the $N$ remaining variables $U_{i}(t)$.

Taking the $U_{i}$-dependent terms on the right-hand side of (2.2) as additional inputs, we first use a variations of parameter formula to yield

$$
\begin{array}{r}
V_{i \alpha}(t)=\sum_{\beta \in \Gamma} G_{\alpha \beta}(t) V_{i \beta}(0) \\
+\int_{0}^{t} d t^{\prime} \sum_{\beta \in \Gamma} G_{\alpha \beta}\left(t-t^{\prime}\right) \\
\times\left[I_{i \beta}\left(t^{\prime}\right)+\hat{\gamma}_{0} U_{i}\left(t^{\prime}\right) \delta_{\beta, \alpha_{0}}\right. \\
\left.\quad+\sum_{j} W_{i j}^{\beta} f\left(U_{j}\left(t^{\prime}\right)\right)\right],
\end{array}
$$


where a factor of $C_{\alpha}$ has been absorbed into $I_{i \alpha}(t)$ and the matrix $\mathbf{G}(t)$ satisfies

$$
\begin{array}{r}
G_{\alpha \beta}(t)=\left[e^{t \mathbf{Q}}\right]_{\alpha \beta}, \quad Q_{\alpha \beta}=-\frac{\delta_{\alpha \beta}}{\tau_{\alpha}}+\sum_{\left\langle\beta^{\prime} ; \alpha\right\rangle} \frac{\delta_{\beta, \beta^{\prime}}}{\tau_{\alpha \beta^{\prime}}}, \\
\alpha, \beta \in \Gamma .
\end{array}
$$

The membrane time constant $\tau_{\alpha}$ and junctional time constant $\tau_{\alpha \beta}$ are defined by

$$
\begin{array}{r}
\frac{1}{\tau_{\alpha}}=\frac{1}{C_{\alpha}}\left[\frac{1}{R_{\alpha}}+\frac{1}{r} \delta_{\alpha, \alpha_{0}}+\sum_{\left\langle\beta^{\prime} \in \Gamma ; \alpha\right\rangle} \frac{1}{R_{\alpha \beta^{\prime}}}\right], \\
\tau_{\alpha \beta}=C_{\alpha} R_{\alpha \beta} .
\end{array}
$$

We also set

$$
\hat{\varepsilon}=\frac{1}{\hat{R} \hat{C}}+\frac{1}{r \hat{C}}, \quad \hat{\gamma}_{0}=\frac{1}{r C_{\alpha_{0}}}, \quad \hat{\gamma}=\frac{1}{r \hat{C}} .
$$

It is convenient at this stage to introduce an additional simplification: each weight $W_{i j}^{\alpha}$ is taken to have the product form

$$
W_{i j}^{\alpha}=W_{i j} w_{\alpha}, \sum_{\alpha} w_{\alpha}=1,
$$

which means that the relative spatial distribution of the input from neuron $j$ across the compartments of neuron $i$ is independent of $i$ and $j$. If Eq. (2.4) with $\alpha=\alpha_{0}$ is now substituted into Eq. (2.3), we obtain $N$ coupled nonlinear Volterra integrodifferential equations for the $U_{i}$ of the form

$$
\begin{aligned}
\frac{d U_{i}}{d t}= & -\hat{\varepsilon} U_{i}(t)+\sum_{j} \hat{W}_{i j} f\left(U_{j}(t)\right) \\
& +\int_{0}^{t}\left[G\left(t-t^{\prime}\right) \sum_{j} W_{i j} f\left(U_{j}\left(t^{\prime}\right)\right)\right. \\
& \left.+H\left(t-t^{\prime}\right) U_{i}\left(t^{\prime}\right)\right] d t^{\prime}+F_{i}(t),
\end{aligned}
$$

with the convolution kernels

$$
\begin{aligned}
& H(t)=\left(\hat{\gamma}_{0} \hat{\gamma}\right) G_{\alpha_{0} \alpha_{0}}(t), \\
& G(t)=\hat{\gamma} \sum_{\beta} w_{\beta} G_{\alpha_{0} \beta}(t),
\end{aligned}
$$

and the effective input (after absorbing $\widehat{C}$ into $\widehat{I}_{i}$ )

$$
\begin{aligned}
F_{i}(t)= & \hat{I}_{i}(t)+\hat{\gamma} \sum_{\beta \in \Gamma} G_{\alpha_{0} \beta}(t) V_{i \beta}(0) \\
& +\hat{\gamma} \int_{0}^{t} \sum_{\beta \in \Gamma} G_{\alpha_{0} \beta}\left(t-t^{\prime}\right) I_{i \beta}\left(t^{\prime}\right) d t^{\prime} .
\end{aligned}
$$

Equation (2.9) is the functional extension of the standard analogue model [13] to the case of neurons with dendritic structure. Note that we recover the standard model in the limit $\gamma, \hat{\gamma} \rightarrow 0$ or $r \rightarrow \infty$. All information concerning the passive membrane properties and topology of the dendrites is represented compactly in terms of the convolution kernels $H(t)$ and $G(t)$, Eqs. (2.10) and (2.11). These, in turn, are given in terms of the functions $G_{\alpha \beta}(t)$ defined in Eq. (2.5). We may identify $G_{\alpha \beta}(t)$ as the compartmental model response function, since $G_{\alpha \beta}(t)$ determines the membrane potential of dendritic compartment $\alpha$ at time $T$ in response to a unit impulse stimulation of compartment $\beta$ at time $T-t$. The matrix $\mathbf{Q}$ of Eq. (2.5) has real, negative, nondegenerate eigenvalues $\lambda_{r}$ reflecting the fact that the dendritic system is described in terms of a passive $R C$ circuit, which is a dissipative system. Thus, one could determine $G_{\alpha \beta}(t)$ by diagonalizing $\mathbf{Q}$ to obtain $G_{\alpha \beta}(t)=\Sigma_{r} c_{\alpha \beta}^{r} e^{-t\left|\lambda_{r}\right|}$. However, such an approach is rather cumbersome, especially in the case of many compartments, and does not lead to simple analytical expressions that are applicable to arbitrary trees and that have an explicit dependence on system parameters.

Recently, Bressloff and Taylor [3] have developed an alternative method for evaluating $\exp (t \mathbf{Q})$, which does lead to simple analytical expressions for the response function $G_{\alpha \beta}(t)$. Such results apply to trees of arbitrary topology provided that each branch of the tree is uniform and certain conditions are imposed on the membrane properties of compartments at the branching nodes and terminals of the tree. Under such assumptions, one can show that $e^{t \mathbf{Q}}=e^{-\varepsilon t} e^{\gamma t \mathbf{P}}$ where $\varepsilon^{-1}$ and $\gamma^{-1}$ are, respectively, a global membrane time constant and a global junctional time constant of the dendritic system, and the matrix $\mathbf{P}$ generates paths along the tree $\Gamma$. In particular, modulo additional constant factors arising from the boundary conditions at terminals and branching nodes, $\left[\mathbf{P}^{m}\right]_{\alpha \beta}$ is equal to the number of possible paths on $\Gamma$ consisting of $m$ steps between the compartments $\alpha$ and $\beta$, where a step is a single jump between neighboring compartments. Thus, the calculation of $G_{\alpha \beta}(t)$ reduces to (i) determining the sum over paths $\left[\mathbf{P}^{m}\right]_{\alpha \beta}$, and then (ii) evaluating the infinite series $\Sigma_{m \geq 0}(t \gamma)^{m}\left[\mathbf{P}^{m}\right]_{\alpha \beta} / m$ ! obtained by expanding $e^{t \gamma \mathbf{P}}$ in powers of $t$. The first step can be performed using results from the theory of random walks along similar lines to Abbott et al. [14].

The simplest example, which forms the building block for more complex topologies, is an infinite chain of identical dendritic compartments labeled $\alpha=0, \pm 1, \pm 2, \ldots$. Under the uniformity conditions $\tau_{\alpha}=\varepsilon^{-1}, \tau_{\alpha \beta}=\dot{\gamma}^{-1}$, for all $\alpha, \beta$, the matrix $Q$ of Eq. (2.5) reduces to $Q_{\alpha \beta}=-\varepsilon \delta_{\alpha \beta}+\gamma P_{\alpha \beta}$, where $\mathbf{P}$ is the generator of paths along the chain, $P_{\alpha \beta}=\delta_{\alpha, \beta+1}+\delta_{\alpha, \beta-1}$. Using a standard result from the theory of random walks, the number of possible paths of $m$ steps between nodes $\alpha$ and $\beta$ is $N_{0}[\alpha, \beta ; m]=m ! /[(m+|\beta-\alpha| / 2) !(|\beta-\alpha| / 2) !] . \quad$ The infinite series of step (ii) can then be summed explicitly with the result that the response function of the chain, denoted by $G_{\alpha \beta}^{(0)}$, is

$$
G_{\alpha \beta}^{(0)}(t)=e^{-\varepsilon t} I_{|\beta-\alpha|}(2 \gamma t),
$$

where $I_{n}$ is a modified Bessel function of integer order $n$. In Ref. [3], this construction is generalized to the case of arbitrary dendritic topologies. We simply quote the result here. Following Abbott, Farhi, and Gutmann [14], we define a trip to be a path on the tree $\Gamma$ with certain restrictions: a trip from $\beta$ to $\alpha$ starts from $\beta$ in either direc- 
tion, but subsequent changes in direction can only occur by reflections at branching nodes and terminals. Provided that certain uniformity conditions are satisfied at terminal nodes and branching nodes of the tree, the response function can be written in the general form

$$
G_{\alpha \beta}(t)=\sum_{\mu} c_{\mu} G_{\alpha+L_{\mu}, \beta}^{(0)}(t),
$$

where the right-hand side involves a summation over all possible trips $\mu$ and $L_{\mu}$ is the length (total number of steps) of a trip. The coefficients $c_{\mu}$ arise from additional factors picked up at terminal and branching nodes, and there are systematic rules for calculating these coefficients [3]. Note that for complex topologies there is an infinite number of possible trips so that we have an infinite sum over $\mu$. However, since $G_{\alpha \beta}^{(0)}(t)$ is a rapidly decreasing function of the separation $|\beta-\alpha|$ along the chain, the series can be truncated to include only a small number of terms.

Note that in dealing with the infinite dendritic systems described above, one must be careful that the sums over $\beta$ in Eqs. (2.11) and (2.12) are finite. This is ensured by the conditions

$$
\sum_{\beta}\left|I_{\beta}\right|<\infty, \quad \sum_{\beta}\left|w_{\beta}\right|<\infty
$$

since the response functions $G_{\alpha \beta}(t)$ are bounded for all $\alpha, \beta$. For future reference, we also note that in the case of a homogeneous network, Eq. (2.9) reduces to a single scalar equation. Homogeneity is obtained by setting $\hat{I}_{i}=\hat{I}, I_{i \alpha}=I_{\alpha}$ and $W_{i j}=W / N, \hat{W}_{i j}=\hat{W} / N$. We also impose the initial condition $V_{i \beta}(0)=V_{\beta}(0)$; this latter condition is not necessary since the initial values $V_{i \beta}(0)$ only give a transient contribution to $F_{i}$, Eq. (2.12), which vanishes in the large $t$ limit. Under these various homogeneity conditions, we have

$$
\frac{d U_{i}}{d t}-\frac{d U_{j}}{d t}=-\hat{\varepsilon}\left(U_{i}-U_{j}\right) .
$$

Hence for large $t, U_{i}(t) \approx U(t)$ for all $i$, where

$$
\begin{aligned}
\frac{d U}{d t}= & -\hat{\varepsilon} U(t)+\hat{W} f(U(t)) \\
& +\int_{0}^{t}\left[W G\left(t-t^{\prime}\right) f\left(U\left(t^{\prime}\right)\right)\right. \\
& \left.\quad+H\left(t-t^{\prime}\right) U\left(t^{\prime}\right)\right] d t^{\prime}+F(t)
\end{aligned}
$$

and

$$
\begin{aligned}
F(t)= & \hat{I}(t)+\hat{\gamma} \sum_{\beta} G_{\alpha_{0} \beta}(t) V_{\beta}(0) \\
& +\hat{\gamma} \int_{0}^{t} \sum_{\beta} G_{\alpha_{0} \beta}\left(t-t^{\prime}\right) I_{\beta}\left(t^{\prime}\right) d t^{\prime} .
\end{aligned}
$$

Hence, the dynamics of a homogeneous network can be described in terms of a single compartmental model neuron with feedback.

\section{DYNAMICS OF NONLINEAR VOLTERRA INTEGRODIFFERENTIAL EQUATIONS}

The existence of a general analytical expression for the response function of an arbitrary dendritic tree means that our functional approach, which reduces $N(M+1)$ coupled ordinary differential equations to $N$ coupled integrodifferential equations, is potentially a powerful technique for studying the dynamics of recurrent compartmental networks. In order to explore this further, we first need to consider some general results concerning the dynamics of nonlinear Volterra integrodifferential equations. For a review of Volterra equations see Ref. [15] and references therein. Another useful reference is Cushing [9], who analyzes a class of nonlinear integrodifferential equations arising in population dynamics.

Consider the nonlinear Volterra integrodifferential equation

$$
\frac{d \mathbf{x}}{d t}=-\mathbf{f}(\mathbf{x}(t))+\int_{t_{0}}^{t} \mathbf{B}\left(t-t^{\prime}\right) \mathbf{g}\left(\mathbf{x}\left(t^{\prime}\right)\right) d s,
$$

where $\mathbf{x} \in \mathbb{R}^{n}, \mathbf{f}, \mathbf{g}: \mathbb{R}^{n} \rightarrow \mathbb{R}^{n}$ are differentiable, bounded functions and $\mathrm{B}$ is an $n \times n$ matrix of real functions continuous on $[0, \infty)$. Moreover, $B_{i j} \in L^{1}[0, \infty)$ for all $i, j$, that is

$$
\int_{0}^{\infty}\left|B_{i j}(t)\right| d t<\infty
$$

Our approach to analyzing Eq. (3.1) will be the standard one of linearizing about a solution (or an equilibrium) and investigating its stability or instability (in the Liapunov sense). It should always be borne in mind that any stability results are in general only local with respect to the solution being considered.

Generally, one is concerned with finding a solution $\mathbf{x}(t)$ of (3.1) for $t \geq 0$ given a continuous initial function $\mathbf{x}^{0}(t)$ defined over the interval $I=\left[t_{0}, 0\right], t_{0} \leq 0$. Note that the compartmental model constructed in Sec. II corresponds to the special case $t_{0}=0$, so that we would only need to specify the initial value $\mathbf{x}(0)=\mathbf{x}^{0}$. Let $\mathbb{R}^{+}$and $\mathbb{R}^{-}$denote the positive and negative reals. If $\hat{\mathbf{x}}(t)$ is a solution of (3.1) on $\mathbb{R}$ then it is called Liapunov stable if given any $\varepsilon>0$ there exists a corresponding $\delta=\delta(\varepsilon)>0$ such that $\left|\hat{x}_{i}(t)-x_{i}^{0}(t)\right| \leq \delta$ for all $t \in I$ and $i$ implies that any solution of (3.1) satisfying $x_{i}(t)=x_{i}^{0}(t)$ for $t \in I$ exists and $\left|\hat{x}_{i}(t)-x_{i}(t)\right| \leq \delta$ for all $t \in \mathbb{R}^{+}$and $i$. If, in addition, there exists a constant $\delta_{0}$ such that $\left|\hat{x}_{i}(t)-x_{i}^{0}(t)\right| \leq \delta_{0}$ for all $t \in I$ and $i$ implies $\left|\hat{x}_{i}(t)-x_{i}(t)\right| \rightarrow 0$ as $t \rightarrow \infty$, then $\hat{\mathbf{x}}(t)$ is said to be asymptotically stable. In the following we shall restrict our discussion to the case $t_{0}=0$.

Suppose that there exists an equilibrium $x^{*}$ of Eq. (3.1), which satisfies

$$
\mathbf{f}\left(\mathbf{x}^{*}\right)=\mathbf{g}\left(\mathbf{x}^{*}\right) \int_{0}^{\infty} \mathbf{B}\left(t^{\prime}\right) d t^{\prime}=\mathbf{g}\left(x^{*}\right) \widetilde{\mathbf{B}}(0),
$$

where $\widetilde{\mathbf{B}}(z)$ is the Laplace transform of $\mathbf{B}(t)$. Linearizing Eq. (3.1) about this equilibrium gives

$$
\frac{d \mathbf{y}}{d t}=-\mathbf{A y}+\int_{0}^{t} \mathbf{C}\left(t-t^{\prime}\right) \mathbf{y}\left(t^{\prime}\right) d t^{\prime}, \quad \mathbf{y}=\mathbf{x}-\mathbf{x}^{*},
$$

where 


$$
\mathbf{A}=\left.D \mathbf{f}\right|_{\mathrm{x}=\mathrm{x}^{*}}, \mathbf{C}(t)=\left.\mathbf{B}(t) D \mathbf{g}\right|_{\mathrm{x}=\mathrm{x}^{*}}
$$

and

$$
[D \mathbf{f}]_{i j}=\frac{\partial f_{i}}{\partial x_{j}},[D \mathbf{g}]_{i j}=\frac{\partial g_{i}}{\partial x_{j}} \text {. }
$$

The linearized system ignores higher order terms in $\mathbf{x}$. In the more general situation where $t_{0}<0$, the linearized equation also neglects a nonhomogeneous forcing term involving the initial conditions over the interval $I$ [9]. Since $\mathbf{A}$ is a constant matrix and the convolution kernel $\mathbf{C}(t)$ is a continuous $n \times n$ matrix whose elements lie in $L^{1}[0, \infty)$, it can be proved [16] that the zero solution of Eq. (3.4) is asymptotically stable if and only if

$$
\Delta(z) \equiv \operatorname{det}\left[z \mathbf{1}_{n}+\mathbf{A}-\widetilde{\mathbf{C}}(z)\right] \neq 0 \text { when } \operatorname{Re} z \geq 0,
$$

where $\widetilde{\mathbf{C}}(z)$ is the Laplace transform of the kernel $\mathbf{C}$ and $\mathbf{1}_{n}$ is the $n \times n$ unit matrix. Condition (3.7) requires that no roots of the so called characteristic function $\Delta(z)$ should lie in the right-hand side of the complex plane. (This condition generalizes the standard asymptotic stability result for an autonomous dynamical system $\dot{\mathbf{y}}=-\mathbf{A y}$, which requires that all eigenvalues of the matrix A have a positive real part.)

It can also be shown that if the stability condition (3.7) is met for the linear system, then the zero solution of the full nonlinear system (3.1) is locally asymptotically stable [16]. On the other hand, if $\Delta(z)$ has at least one root in the right-half complex plane $(\operatorname{Re} z>0)$ then the linear system (3.5) is unstable [17]. The corresponding instability of the full nonlinear system (3.1) is harder to establish. However, we are interested in the special case $t_{0}=0$, for which more concrete results concerning instability can be established, in particular, a version of the Hopf bifurcation theorem (see Sec. V).

\section{LINEAR STABILITY ANALYSIS OF A COMPARTMENTAL NETWORK}

Let us return to the compartmental model neural network constructed in Sec. 2, in particular, the integrodifferential Eq. (2.9) describing the dynamics of the somatic membrane potentials $U_{i}$. Suppose that the effective input $F_{i}$, Eq. (2.12), is zero. In other words, assume that there are no external inputs and that the transient contribution from the initial values $\mathbf{V}(0)$ is negligible. If the term involving the kernel $H$ on the right-hand side of Eq. (2.9) is ignored ( $\hat{\gamma}_{0} \hat{\gamma}$ sufficiently small), then Eq. (2.9) becomes

$$
\begin{aligned}
\frac{d U_{i}}{d t}= & -\hat{\varepsilon} U_{i}(t)+\sum_{j} \hat{W}_{i j} f\left(U_{j}(t)\right) \\
& +\int_{0}^{t}\left[G\left(t-t^{\prime}\right) \sum_{j} W_{i j} f\left(U_{j}\left(t^{\prime}\right)\right)\right] d t^{\prime},
\end{aligned}
$$

which is identical in form to that of Eq. (3.1) with $x_{i} \rightarrow U_{i}, i=1, \ldots, N$ and

$$
\begin{aligned}
& f_{i}(\mathbf{U})=\hat{\varepsilon} U_{i}-\sum_{j} \hat{W}_{i j} f\left(U_{j}\right), g_{i}(\mathbf{U})=f\left(U_{i}\right), \\
& B_{i j}(t)=W_{i j} G(t) .
\end{aligned}
$$

The particular functions $f_{i}$ and $g_{i}$ of Eq. (4.2) satisfy $f_{i}(\mathbf{0})=g_{i}(\mathbf{0})=0$ so that one solution to $(4.1)$ is the zero solution $\mathbf{U}(t)=\mathbf{0}$. For sake of concreteness, we shall consider linearization about this zero solution. The characteristic function is given by

$$
\Delta(z)=\operatorname{det}\left[(z+\hat{\varepsilon}) \mathbf{I}_{N}-\kappa \hat{\mathbf{W}}-\kappa \mathbf{W} \widetilde{G}(z)\right],
$$

where $f^{\prime}(\mathbf{0})=\kappa$ and $\widetilde{G}(z)$ is obtained from Eq. (2.11),

$$
\widetilde{G}(z)=\hat{\gamma} \sum_{\beta} w_{\beta} \widetilde{G}_{\alpha_{0} \beta}(z) .
$$

If the $H$ term is included on the right-hand side of Eq. (4.1) then the characteristic function is modified such that $z+\hat{\varepsilon}$ is replaced by $z+\hat{\varepsilon}-\widetilde{H}(z)$.

Equations (4.3) and (4.4) show how the stability of the network depends on the interneuron connection weights $W_{i j}, \hat{W}_{i j}$, the distribution of inputs across the compartments of each neuron as specified by the $w_{\alpha}$, and the internal structure of the individual neurons as represented by the Laplace transformed response function $\widetilde{G}_{\alpha_{0} \beta}$. This in turn highlights the usefulness of the construction of the compartmental response function developed by Bressloff and Taylor [3]. For using Eq. (2.14) and the fact that the Laplace transformation is a linear operation, we can determine $\widetilde{G}_{\alpha_{0} \beta}(z)$ for an arbitrary tree in terms of a linear sum involving the Laplace transformed response function of a uniform, infinite dendritic chain. The latter can be calculated explicitly using properties of the modified Bessel function [Eq. (2.13)]. The result is [6]

$$
\widetilde{G}_{\alpha \beta}^{(0)}(z)=\frac{1}{\gamma} \frac{\lambda_{-}(z)^{|\alpha-\beta|}}{\lambda_{+}(z)-\lambda_{-}(z)}
$$

with

$$
\lambda_{ \pm}(z)=\frac{(z+\varepsilon)}{2 \gamma} \pm\left[\left(\frac{(z+\varepsilon)}{2 \gamma}\right]^{2}-1\right]^{1 / 2} .
$$

In order to simplify the analysis, we shall assume that there is no direct input stimulation of the soma, that is, $\hat{W}_{i j}=0$. Then we can proceed by diagonalizing the connection matrix $\mathbf{W}$ to obtain the set of characteristic equations

$$
z+\hat{\varepsilon}-\kappa W_{i} \widetilde{G}(z)=0,
$$

where $W_{i}, i=1, \ldots, N$, is an eigenvalue of $\mathbf{W}$. The zero solution is (locally) asymptotically stable if and only if for each eigenvalue $W_{i}$ there are no solutions to Eq. (4.7) that lie in the right-half complex plane. A necessary condition for asymptotic stability is expressed by the following theorem (cf. Ref. [9]).

Theorem 1: The zero solution is (locally) asymptotically stable if

$$
\kappa\left|W_{i}\right|<\hat{\varepsilon} / \widetilde{G}(0), \quad i=1, \ldots, N .
$$


Proof: Since $G(t) \in L^{1}[0, \infty)$, the Laplace transform $\widetilde{G}(z)$ exists for $\operatorname{Re} z \geq 0$ such that $\widetilde{G}(z) \leq \widetilde{G}(0)$. Suppose that Eq. (4.8) holds. Then $|z+\hat{\varepsilon}| \geq \hat{\varepsilon}>\kappa\left|W_{i}\right| \widetilde{G}(0)$ $\geq\left|\kappa W_{i} \widetilde{G}(z)\right|$ for $\operatorname{Re} z \geq 0$, and $i=1, \ldots, N$ which implies that $\Delta(z)$ cannot vanish for $\operatorname{Re} z \geq 0$, i.e., there are no roots in the right-half complex plane. Hence, the system is asymptotically stable about the zero solution.

In the case of a real eigenvalues $W$, when $W$ increases beyond the positive value $\hat{\varepsilon} / \kappa \widetilde{G}(0)$ a real root crosses the imaginary axis leading to a destabilization of the zero solution (via a pitchfork bifurcation). For if $\kappa W>\hat{\varepsilon} / \widetilde{G}(0)$, then $\Delta(0)<0$, whereas $\Delta(x) \rightarrow \infty$ as $x \rightarrow \infty, x$ real [since $\widetilde{G}(z)$ is bounded for $\operatorname{Re} z \geq 0$ ]. This means that there exists a positive real root of $\Delta(z)$ and thus the system is unstable.

We are interested in determining the conditions for marginal stability in which a pair of complex roots $\pm i \omega$ cross the imaginary axis, which is a prerequisite for a Hopf bifurcation. It is useful to write the characteristic Eq. (4.7) for pure imaginary roots and a given complex eigenvalue $W=W^{\prime}+i W^{\prime \prime}$ in the form

$i \omega+\hat{\varepsilon}-\kappa\left(W^{\prime}+i W^{\prime \prime}\right) \int_{0}^{\infty} e^{-i \omega t} G(t) d t=0, \omega$ real .

Equating real and imaginary parts of Eq. (4.9) then yields (after some simple algebra)

$W^{\prime}=\kappa^{-1}[\widehat{\varepsilon} C(\omega)-\omega S(\omega)] /\left[C(\omega)^{2}+S(\omega)^{2}\right]$,

$W^{\prime \prime}=\kappa^{-1}[\hat{\varepsilon} S(\omega)+\omega C(\omega)] /\left[C(\omega)^{2}+S(\omega)^{2}\right]$,

with

$$
\begin{aligned}
& S(\omega)=\int_{0}^{\infty} \sin (\omega t) G(t) d t, \\
& C(\omega)=\int_{0}^{\infty} \cos (\omega t) G(t) d t .
\end{aligned}
$$

By definition, $C(\omega)=\operatorname{Re} \widetilde{G}(i \omega)$ and $S(\omega)=-\operatorname{Im} \widetilde{G}(i \omega)$.

The linear stability analysis can now be developed along similar lines to that of Marcus and Westervelt [10]. For example, the stability region in the complex $W$ plane is obtained by finding for each angle $\theta=\tan ^{-1}\left(W^{\prime \prime} / W^{\prime}\right)$ the solution $\omega$ of Eq. (4.10) corresponding to the smallest value of $|W|$. Other roots of (4.10) produce larger values of $|W|$, which lie outside the stability region defined by $\omega$. (The existence of such a region is ensured by theorem 1.) To illustrate the basic idea we shall determine the stability region for the two generic kernels (Cushing, 1977) (a) $G(t)=T^{-1} \exp (-t / T)$ and (b) $G(t)=T^{-2} t \exp (-t / T)$. The first kernel qualitatively represents a "weak" delay in the sense that the maximum response at time $t$ is due to the current input stimulation. On the other hand, the second kernel qualitatively represents a "strong" delay since the maximum response at time $t$ is due to an input stimulation at a previous time $t-T$, i.e., the maximum of the response curve occurs at $t=T$. For these so called generic kernels one can obtain explicit solutions to Eq. (4.10). Moreover, although they are not themselves compartmental response functions they do capture the qualitative behavior of the latter. This point is illustrated in Fig. 2 where the response function $G_{0 \alpha}^{(0)}(t)$ of a uniform, infinite dendritic chain [Eq. (2.13)] is plotted for a range

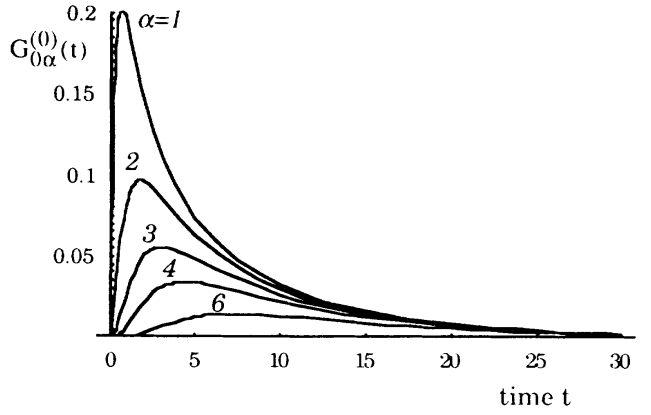

FIG. 2. Response function $G_{0 \alpha}^{(0)}(t)$ of an infinite, uniform dendritic chain as a function of $t$ (in units of $\gamma^{-1}$ ) with $\varepsilon=1.1 \gamma$ for various values of the separation $\alpha$.

of values of the separation $|\alpha|$. We see that the $\alpha=0$ curve corresponds to a weak delay, whereas the response curves for $|\alpha|>0$ correspond to strong delays with the time of maximum response $T$ increasing with $|\alpha|$.

(a) Weak delay. Substitution of $G(t)=T^{-1} \exp (-t / T)$ into Eq. (4.11) gives

$$
S(\omega)=\frac{\omega T}{1+(\omega T)^{2}}, \quad C(\omega)=\frac{1}{1+(\omega T)^{2}} .
$$

From Eqs. (4.10) and (4.12), we find that the boundary curve of the stability region is given by the parabola (Fig. 3)

$$
\kappa W^{\prime}=\hat{\varepsilon}-\frac{T\left(\kappa W^{\prime \prime}\right)^{2}}{(1+\hat{\varepsilon} T)^{2}}
$$

and the corresponding value of the imaginary root is $\omega=W^{\prime \prime} /(1+\hat{\varepsilon} T)$. It follows that for real eigenvalues $\left(W^{\prime \prime}=0\right)$ there are no pure imaginary roots of the characteristic equation (4.9). Thus, for a connection matrix W

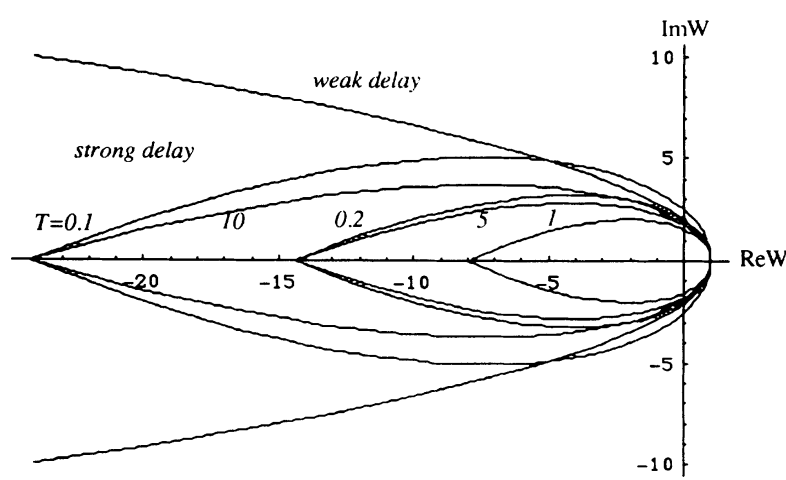

FIG. 3. Stability region in complex $W$ plane of the origin for a recurrent network with generic delay kernels, where $W$ is an eigenvalue of the interneuron connection matrix $\mathbf{W}$. For a weak delay kernel, the stability region is open with the boundary curve given by a parabola. On the other hand, the stability region is closed in the strong delay case with the boundary curve crossing the real axis in the negative half plane at a $T$-dependent value $W^{-}$. This is shown for various values of the delay $T$ with the delay rate $\hat{\varepsilon}$ and gain $\kappa$ both set to unity. All boundary curves meet on the positive half of the real axis at the same point $W^{+}=\hat{\varepsilon} / \kappa=1$. 
that only has real roots (e.g., a symmetric matrix), destabilization of the zero solution occurs when the largest positive eigenvalue increases beyond the value $\hat{\varepsilon} / \kappa$, and this corresponds to a real root of the characteristic equation crossing the imaginary axis. Hence, oscillations cannot occur via a Hopf bifurcation.

(b) Strong delay. Substitution of $G(t)$ $=T^{-2} t \exp (-t / T)$ into Eq. (4.11) gives

$$
S(\omega)=\frac{2 \omega T}{\left[1+(\omega T)^{2}\right]^{2}}, \quad C(\omega)=\frac{1-\omega^{2} T^{2}}{\left[1+(\omega T)^{2}\right]^{2}} .
$$

The solution of Eq. (4.10) is

$$
\begin{aligned}
& \kappa W^{\prime}=\widehat{\varepsilon}\left(1-\omega^{2} T^{2}\right)-2 \omega^{2} T, \\
& \kappa W^{\prime \prime}=2 \hat{\varepsilon} \omega T+\omega\left(1-\omega^{2} T^{2}\right) .
\end{aligned}
$$

Equation (4.15) defines a parametric curve in the complex $W$ plane, and the boundary of the stability region is the closed portion of this curve as shown in Fig. 3 for a range of delays $T$. Since the stability region closes in the lefthalf plane, it is now possible for the origin to lose stability when the largest negative eigenvalue crosses the boundary, even when this eigenvalue is real. Whether or not this leads to a Hopf bifurcation can then be explored using the analysis of Sec. 5. Note from Eq. (4.15) that the points of destabilization on the positive and negative real $W$ axis are given, respectively, by $W^{+}=\hat{\varepsilon} / \kappa$ and $W^{-}=-\left(4 \hat{\varepsilon}+2 T^{-1}+2 \hat{\varepsilon}^{2} T\right) / \kappa$. Thus, $W^{+}$is independent of the delay $T$, whereas $W^{-}$has the $T$ dependence shown in Fig. 4. The minimum value of $\boldsymbol{W}^{-}$occurs at $T=1 / \hat{\varepsilon}$. There is consequently a major difference in the behavior of the stability region as a function of the delay $T$ compared to the case of discrete delays analyzed in Ref. [10]. In the latter problem, the stability region shrinks as the discrete delay $T$ increases approaching a circle of radius proportional to $1 / \kappa$ in the limit $T \rightarrow \infty$. On the other hand, in the continuous case considered here the stability region shrinks as $T$ increases until it reaches the value $1 / \hat{\varepsilon}$, and thereafter increases with $T$.

To illustrate how the results for the generic delay kernel relate to those obtained using a compartmental response function, we shall determine the point of intersection $\mathrm{W}^{-}$of the stability region boundary with the negative real axis for an infinite dendritic chain of identical compartments labeled $\alpha=0, \pm 1, \pm 2, \ldots$ (Sec. II). Let the

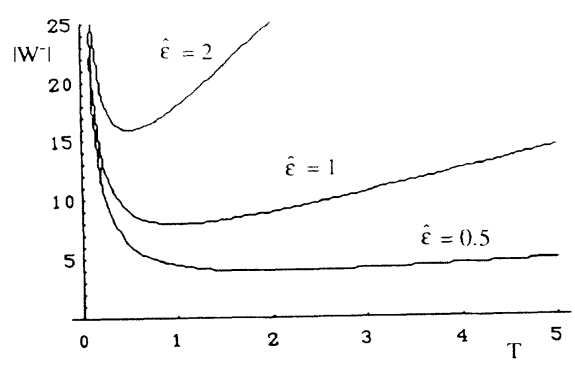

FIG. 4. Plot of the intercept $W^{-}$of the stability region of the strong generic delay kernel as a function of the delay $T$. Various values of $\hat{\varepsilon}$ are considered with $\kappa=1$. The minimum value of $W^{-}$occurs at $T=1 / \hat{\varepsilon}$. dendritic compartment immediately adjoining the soma be $\alpha_{0}=0$ and assume for simplicity that $w_{\beta}=\delta_{\beta, L}$ for some $L$. Equation (2.11) then implies that $G(t)=G_{0, L}^{(0)}(t)$. Setting $W^{\prime \prime}=0$ in Eq. (4.10) shows that

$$
W^{-}=\hat{\varepsilon} / \kappa C(\omega),
$$

where $\omega$ is the smallest nonzero solution (assuming it exists) to the equation

$$
\omega=-\hat{\varepsilon} T_{L}(\omega), \quad T_{L}(\omega)=\frac{S_{L}(\omega)}{C_{L}(\omega)} .
$$

The dependence on the separation $L$ has been made explicit. Equations (4.5), (4.6), and (4.14) imply that

$$
C_{L}(\omega)-i S_{L}(\omega)=\frac{1}{2 \gamma} \frac{\left[\varepsilon+i \omega-\sqrt{(\varepsilon+i \omega)^{2}-4 \gamma^{2}}\right]^{L}}{\sqrt{(\varepsilon+i \omega)^{2}-4 \gamma^{2}}}
$$

An iterative equation for $T_{L}(\omega)$ can be obtained using the identity

$$
\sqrt{(\varepsilon+i \omega)^{2}-4 \gamma^{2}}=A+i B
$$

where

$$
\begin{aligned}
& A^{2}=\frac{1}{2}\left[f(\omega)+\sqrt{f(\omega)^{2}+4 \omega^{2} \varepsilon^{2}}\right], \\
& B^{2}=\frac{1}{2}\left[-f(\omega)+\sqrt{f(\omega)^{2}+4 \omega^{2} \varepsilon^{2}}\right], \\
& f(\omega)=\varepsilon^{2}-4 \gamma^{2}-\omega^{2} .
\end{aligned}
$$

First, substituting Eq. (4.19) into (4.18) for $L=0$ gives

$$
T_{0}(\omega)=\frac{B}{A} \text {. }
$$

Second, writing Eq. (4.18) for $L \geq 1$ in the form

$$
\begin{aligned}
C_{L}(\omega) & -i S_{L}(\omega) \\
= & {[(\varepsilon-A)+i(\omega-B)]\left[C_{L-1}(\omega)-i S_{L-1}(\omega)\right] }
\end{aligned}
$$

and equating real and imaginary parts leads to the result

$$
T_{L}(\omega)=\frac{(\varepsilon-A) T_{L-1}(\omega)+B-\omega}{(\omega-B) T_{L-1}(\omega)+\varepsilon-A} .
$$

The point of intersection $W^{-}$can now be obtained for a range of $L$ values using equations (4.16), (4.17), (4.20), (4.21), and (4.23). The results are displayed in Fig. 5 for $\hat{\varepsilon}=1=\kappa$. As expected, $W^{-}$does not exist for $L=0$ since $G_{00}^{(0)}(t)$ is equivalent to a weak delay. On the other hand, for $L>0, G_{0, L}^{(0)}(t)$ is equivalent to a strong delay with the time of maximum response a monotonically increasing function of the separation $L$ (Fig. 2). Thus comparison of Figs. 4 and 5 shows that the compartmental response function exhibits the same qualitative behavior as the generic kernel. 


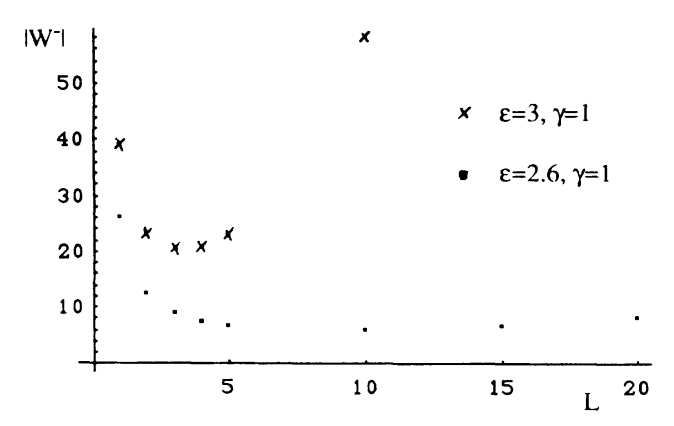

FIG. 5. Plot of the intercept $W^{-}$of the stability region of compartmental model neural network with kernel $G_{0 L}^{(0)}(t)$ as a function of the separation $L$.

\section{ONSET OF OSCILLATIONS IN A COMPARTMENTAL MODEL NETWORK}

Let $\mu$ be some real parameter of the dynamical system described by Eq. (4.1). For example, $\mu$ could correspond to one of the weights of the network or to a membrane potential time constant. It may happen that the zero solution (or some other equilibrium) is stable for $\mu<\mu_{0}$, but that as $\mu$ increases above $\mu_{0}$, a pair of zeros of the characteristic function $\Delta(z)$ cross the imaginary axis resulting in a destabilization of the equilibrium for $\mu>\mu_{0}$. Using the transfer function approach of Allwright [12], we shall derive conditions under which this destabilization is associated with the appearance or disappearance of a periodic solution via a Hopf bifurcation. Note that there are two distinct scenarios here: either the periodic solutions are stable and exist when the equilibrium is unstable (supercritical Hopf bifurcation), or the periodic solutions are unstable and exist when the equilibrium is stable (subcritical Hopf bifurcation).

In order to proceed, we first need to express Eq. (4.1) in a particular form. Define a vector $\hat{U}(t) \in \mathbb{R}^{N}$ according to the integral equation

$$
\widehat{U}_{i}(t)=\int_{0}^{t} G\left(t-t^{\prime}\right) \sum_{j} W_{i j} f\left(U_{j}\left(t^{\prime}\right)\right) d t^{\prime},
$$

so that Eq. (4.1) becomes

$$
\frac{d U_{i}}{d t}=-\hat{\varepsilon} U_{i}(t)+\sum_{j} \hat{W}_{i j} f\left(U_{j}(t)\right)+\hat{U}_{i}(t)
$$

which can be formally integrated to yield

$U_{i}(t)=\int_{0}^{t} e^{-\varepsilon\left(t-t^{\prime}\right)}\left[\widehat{U}_{i}\left(t^{\prime}\right)+\sum_{j} \hat{W}_{i j} f\left(U_{j}\left(t^{\prime}\right)\right)\right] d t^{\prime}$.

[Since $\hat{\varepsilon}>0$, we can drop the resulting transient contribution to Eq. (5.3) involving the initial condition $U(0)$.] Thus, the $N$-dimensional Volterra integrodifferential equation of (4.1) is equivalent to a $2 N$-dimensional Volterra integral equation of the second kind obtained by combining Eqs. (5.1) and (5.3) to give

$$
\Psi(t)=\int_{0}^{t} \mathcal{S}\left(t-t^{\prime}\right) \mathcal{F}\left(\Psi\left(t^{\prime}\right)\right) d t^{\prime}
$$

with

$$
\begin{aligned}
& \Psi(t)=\left[\begin{array}{l}
\mathbf{U}(t) \\
\hat{\mathbf{U}}(t)
\end{array}\right], \quad \mathcal{G}(t)=\left[\begin{array}{cc}
\mathbf{0} & e^{-\hat{\varepsilon} t} \mathbf{1} \\
\mathbf{B}(t) & \mathbf{0}
\end{array}\right], \\
& \mathscr{F}(\Psi)=\left[\begin{array}{c}
\mathbf{g}(\mathbf{U}) \\
\hat{\mathbf{U}}-\mathbf{f}(\mathbf{U})+\hat{\varepsilon} \mathbf{U}
\end{array}\right],
\end{aligned}
$$

and $B, f, g$ defined in Eq. (4.2). Equation (5.4), or its Laplace transform $\widetilde{\Psi}(z)=\mathscr{S}(z) \mathcal{L}[\mathcal{F}(\Psi)](z)$ can be represented by the simple feedback system of Fig. 6. This consists of a linear transfer function $\mathcal{S}$ and nonlinear feedback $\mathcal{F}$. The onset of oscillations in feedback systems of this form can be analyzed using the Hopf bifurcation theorem of Allwright [12]. We shall present the general theorem and then discuss it for $\mathcal{S}$ and $\mathscr{F}$ satisfying Eq. (5.5), which corresponds to the particular case of a system described by the nonlinear Volterra integrodifferential Eq. (4.1).

Hopf Bifurcation theorem [12]: Consider an equation of the form (5.4) with $\Psi \in \mathbb{R}^{m}$ such that the mapping $\mathcal{F}: \mathbb{R}^{m} \rightarrow \mathbb{R}^{m}$ is parametrized by $\mu$ and is $C^{4}$ jointly with respect to $\Psi$ and $\mu$. (One can weaken this differentiability requirement.) Let $\widetilde{\mathcal{S}}(z)$, which is the Laplace transform of $S(t)$, be $C^{1}$ with respect to $(\mu, z)$. Suppose that there is an equilibrium $\Psi=\Psi^{*}$ where $\Psi^{*}=\widetilde{\mathscr{S}}(0) \mathcal{H}\left(\Psi^{*}\right)$. Define the Jacobian $J=D \mathscr{H}\left(\Psi^{*}\right)$, the tensors $\mathbf{K}=D^{2} \mathcal{F}\left(\Psi^{*}\right), \mathbf{L}=D^{3} \mathcal{F}\left(\Psi^{*}\right)$, and the characteristic function $\Xi(z)=\operatorname{det}\left[1_{m}-\widetilde{\mathscr{S}}(z) J\right]$. Assume that the following conditions hold.

(a) The characteristic function $\Xi(z)$ has a single pair of zeros that cross the imaginary axis with values $\pm i \omega_{0}$ at $\mu=\mu_{0}$. More precisely, let $\mathbf{u}$ and $\mathbf{v}$ be left and right eigenvectors associated with the local continuation of the eigenvalue $i \omega_{0}$, that is, $\left[\mathbf{1}_{m}-\widetilde{\mathscr{S}}(z) \mathbf{J}\right] \mathbf{v}=\lambda(z) \mathbf{v}$ and $\mathbf{u}^{t}\left[\mathbf{1}_{m}-\widetilde{\mathscr{S}}(z) \mathbf{J}\right]=\lambda(z) \mathbf{u}^{t}$ with $\lambda\left(i \omega_{0}\right)=0$. Then we assume that $\mathbf{u}^{t} \widetilde{\mathcal{Q}}^{\prime}(z) \mathbf{J} \mathbf{v} \neq 0$ at $\left(\mu_{0}, i \omega_{0}\right)$, where the prime indicates differentiation with respect to $z$, so that there is a local $C^{1}$ solution $\hat{z}$ of $\Xi(\hat{z})=0$, reducing to $i \omega_{0}$ at $\mu_{0}$.

(b) At the bifurcation point $\mu=\mu_{0}, \Xi(0) \neq 0$. This ensures that the equilibrium is locally $C^{1}$ in $\mu$.

(c) There are no bifurcations at any integer multiples of $i \omega_{0}$, that is, $\Xi\left(i p \omega_{0}\right) \neq 0$ for all integers $p \geq 2$.

(d) The local solution $\hat{z}$ and curvature $\sigma$ [defined below by Eq. (5.6)] satisfy

$$
\operatorname{Re}\left(\frac{d \hat{z}}{d \mu}\right) \neq 0 \text { at } \mu_{0}, \quad \operatorname{Re}(\sigma) \neq 0 \text { at } \mu_{0}, \omega_{0}
$$

Then for $\mu=\mu_{0}+\chi \delta^{2}$, where $\chi= \pm 1$ and $\delta>0$ sufficiently small, there are nonconstant periodic solutions of (5.4) of the form

$$
\Psi(t)=\Psi^{*}+\operatorname{Re} \sum_{k=0}^{2} a_{k} e^{i k \omega t}+O\left(\delta^{3}\right),
$$

with 


$$
\begin{aligned}
& \theta^{2}=\chi \delta^{2} \frac{\operatorname{Re}[d \hat{z} / d \mu]}{\operatorname{Re}[\sigma]}, \quad \chi=\operatorname{sgn} \frac{\operatorname{Re}[d \hat{z} / d \mu]}{\operatorname{Re}[\sigma]}, \\
& \omega=\omega_{0}+\chi \theta^{2}+O\left(\delta^{3}\right), \\
& \mathbf{a}_{0}=\theta^{2} \mathbf{v}_{0}, \mathbf{v}_{0}=\mathscr{H}(0) \mathbf{K}(\mathbf{v} \otimes \overline{\mathbf{v}}) / 4, \\
& \mathbf{a}_{1}=\theta \mathbf{v}, \\
& \mathbf{a}_{2}=\theta^{2} \mathbf{v}_{2}, \quad \mathbf{v}_{2}=\mathcal{H}(2 i \omega) \mathbf{K}(\mathbf{v} \otimes \mathbf{v}) / 4,
\end{aligned}
$$

and

$$
\mathcal{H}(z)=\left[\mathbf{1}_{m}-\mathcal{S}(z) \mathbf{J}\right]^{-1} \mathcal{G}(z) .
$$

[Here " $\otimes$ " is the tensor product so that, for example, $\mathbf{K}(\mathbf{u} \otimes \mathbf{v})$ is a vector with components $\sum_{j, k} K_{i j k} u_{j} v_{k}$.] Furthermore, if we assume that all other zeros of $\Xi(z)$ have negative real parts bounded uniformly away from zero in some neighborhood of $\mu_{0}$, and that $\operatorname{Re}(d \hat{z} / d \mu)>0$, then $\Psi^{*}$ is locally stable for $\mu<\mu_{0}$ and unstable for $\mu>\mu_{0}$. It follows that $\chi>0$ or $\operatorname{Re}(\sigma)>0$ is a sufficient condition for a supercritical Hopf bifurcation in which a stable limit cycle appears as $\mu$ increases beyond $\mu_{0}$.

We have yet to write down the explicit expression for the curvature $\sigma$. This is given by

$$
\sigma=\frac{\mathbf{u}^{t} \mathcal{S}(i \omega) \hat{\mathbf{v}}}{\mathbf{u}^{t} \mathcal{S}^{\prime}(i \omega) \mathbf{J} \mathbf{v}}
$$

with

$$
\widehat{\mathbf{v}}=\mathbf{L}(\mathbf{v} \otimes \mathbf{v} \otimes \overline{\mathbf{v}}) / 8+\mathbf{K}\left(\mathbf{v} \otimes \mathbf{v}_{0}+\overline{\mathbf{v}} \otimes \mathbf{v}_{2} / 2\right) .
$$

Note that $\mathbf{u}$ and $\mathbf{v}$ appearing in Eq. (5.6) are to be evaluated at $z=i \omega$, so $\sigma$ is a continuous function of $(\mu, \omega)$. This concludes the statement of the theorem.

We now apply the above Hopf bifurcation theorem to the nonlinear Volterra integrodifferential equation of (4.1), which has the block diagram representation of Fig. 6 with convolution kernel $\mathcal{S}$ and nonlinear function $\mathcal{F}$ satisfying equation (5.5), and $m=2 N$. We linearize about the zero solution and use $f^{\prime}(0)=\kappa, f^{\prime \prime}(0)$ $=0, f^{\prime \prime \prime}(0)=-2 \kappa^{3}$. Equations (4.2) and (5.5) imply that the Laplace transform $\widetilde{\mathcal{S}}$ and Jacobian $\mathbf{J}=D \mathscr{F}(0)$ have the matrix form

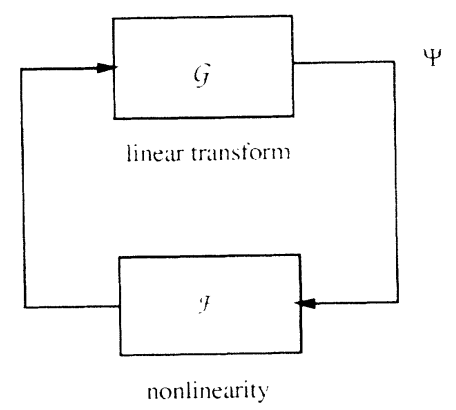

FIG. 6. Block diagram representation of a recurrent compartmental model network.
$\widetilde{S}(z)=\left[\begin{array}{cc}\mathbf{0} & (z+\hat{\varepsilon})^{-1} \mathbf{1} \\ \mathbf{W} \widetilde{G}(z) & \mathbf{0}\end{array}\right], \quad \mathbf{J}=\left(\begin{array}{cc}\kappa \mathbf{1} & 0 \\ \kappa \hat{\mathbf{W}} & \mathbf{1}\end{array}\right]$,

where each block is an $N \times N$ matrix. Moreover, $\mathbf{K}=\mathbf{0}$ and the only nonzero components of the tensor $\mathbf{L}=D^{3} \mathfrak{F}(0)$ are

$L_{i j k l}=-2 \kappa^{3} \delta_{i, j} \delta_{i, k} \delta_{i, l}$,

$L_{i+N, j k l}=-2 \kappa^{3} \hat{W}_{i j} \delta_{j, k} \delta_{j, l}, \quad i, j, k, l=1, \ldots, N$.

Equation (5.8) shows that the characteristic function $\Xi(z)$ is equivalent to the characteristic function $\Delta(z)$ of Eq. (4.3), that is, $\Xi(z)=\Delta(z) /(z+\hat{\varepsilon})$. Equations (5.8) and (5.9) show that the curvature $\sigma$ takes the explicit form $\sigma=\sigma_{1} / \sigma_{2}$ with

$$
\sigma_{1}=(i \omega+\hat{\varepsilon})^{-1} \sum_{i=1}^{N} u_{i} \widehat{v}_{i+N}+\widetilde{G}(i \omega) \sum_{i, j=1}^{N} u_{i+N} W_{i j} \widehat{v}_{j}
$$

and

$$
\begin{aligned}
\sigma_{2}= & -(i \omega+\hat{\varepsilon})^{-2} \sum_{i, j=1}^{N}\left(u_{i} \delta_{i j} v_{j+N}+\kappa u_{i} \hat{W}_{i j} v_{j}\right) \\
& +\kappa \widetilde{G}^{\prime}(i \omega) \sum_{i, j=1}^{N} u_{i+N} \boldsymbol{W}_{i j} v_{j}
\end{aligned}
$$

where

$$
\hat{v}_{i}=-\kappa^{3} v_{i} v_{i} \bar{v}_{i} / 4, \quad \hat{v}_{i+N}=-\kappa^{3} \sum_{j} W_{i j} v_{j} v_{j} \bar{v}_{j} / 4
$$

Note that the linear contribution from the kernel $H$ [Eq. (2.9)] is easily included in the above analysis by replacing $z+\hat{\varepsilon}$ with $z+\hat{\varepsilon}-\widetilde{H}(z)$.

Example (i): To illustrate the application of the Hopf bifurcation theorem, we shall consider the simple case of a single neuron with feedback and one dendritic compartment, which also describes the coherent state of a homogeneous network (Fig. 7). (Such an example can of course be studied more simply using planar analysis.) We shall assume that the feedback excites the somatic compartment and inhibits the dendritic compartment. Moreover, the two compartments are taken to have the same membrane properties. All external inputs are set to zero. Let $V$ and $U$ denote, respectively, the dendritic and somatic membrane potentials. An application of Kirchoff's law yields the pair of differential equations [cf. Eqs. (2.2) and (2.3)]

$$
\begin{aligned}
& \frac{d V}{d t}=-\varepsilon V+\gamma U-W f(U), \quad \varepsilon>\gamma \\
& \frac{d U}{d t}=-\varepsilon U+\gamma V+\hat{W} f(U) .
\end{aligned}
$$

In this simple system we can reexpress Eqs. (5.13) and (5.14) in the block diagram form of Fig. 6 by direct in- 


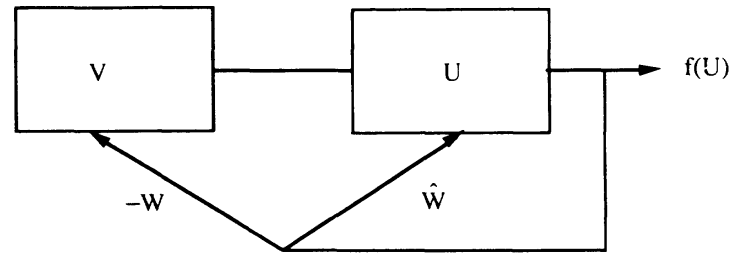

FIG. 7. Schematic diagram of a neuron with excitatory feedback $(\hat{W})$ to the soma and inhibitory feedback $(-W)$ to a single dendritic compartment. The somatic potential is $U$ and the dendritic potential is $V$. Such a system can exhibit oscillatory behavior via a supercritical Hopf bifurcation.

tegration rather than using the procedure described by Eq. (5.1) onwards. The result is Eq. (5.4) with

$$
\begin{aligned}
& \Psi(t)=\left[\begin{array}{l}
U(t) \\
V(t)
\end{array}\right], \quad \mathcal{S}(t)=\left[\begin{array}{cc}
0 & e^{-\varepsilon t} \\
e^{-\varepsilon t} & 0
\end{array}\right], \\
& \mathcal{F}(\Psi)=\left[\begin{array}{l}
\gamma U-W f(U) \\
\gamma V+\hat{W} f(U)
\end{array}\right] .
\end{aligned}
$$

Linearization about the equilibrium $(U, V)=(0,0)$ gives the Jacobian

$$
\mathbf{J}=\left[\begin{array}{cc}
\gamma-\kappa W & 0 \\
\kappa \hat{W} & \gamma
\end{array}\right]
$$

The characteristic function $\Delta(z)$ is

$$
\Delta(z)=z+\varepsilon-\kappa \hat{W}-\frac{\gamma}{z+\varepsilon}(\gamma-\kappa W),
$$

which has a pair of zeros

$$
\lambda_{ \pm}=-\varepsilon+\kappa \hat{W} / 2 \pm \sqrt{(\kappa \hat{W} / 2)^{2}+\gamma^{2}-\gamma \kappa W} .
$$

It follows that the condition of marginal stability with a pair of imaginary eigenvalues $\lambda_{ \pm}= \pm i \omega_{0}$ is

$$
\kappa \hat{W}=2 \varepsilon, \varepsilon^{2}+\gamma^{2}<\gamma \kappa W .
$$

In other words, $\Delta\left( \pm i \omega_{0}\right)=0$ when $\hat{W}=2 \varepsilon / \kappa$, where $\omega_{0}=\sqrt{\gamma \kappa W-\varepsilon^{2}-\gamma^{2}}$. Note that pure imaginary eigenvalues can only exist if the feedback to the dendritic compartment is inhibitory. Assume that $W$ is sufficiently large so that the second condition of (5.19) is satisfied, and take the bifurcation parameter to be $\mu=\hat{W}$. The critical point is then $\mu_{0}=2 \varepsilon / \kappa$. For $\mu<\mu_{0}$ the equilibrium is locally asymptotically stable, whereas it is unstable when $\mu>\mu_{0}$.

The main step in the application of the Hopf bifurcation theorem is the determination of the curvature $\sigma$ of Eq. (5.6). This in turn requires evaluating the Jacobian $\mathbf{J}=\boldsymbol{D} \mathfrak{F}(0)$, the tensors $\mathbf{K}=D^{2} \mathfrak{F}(0)$ and $\mathbf{L}=D^{3} \mathcal{F}(0)$, and the left and right eigenvectors $\mathbf{u}\left(\omega_{0}\right), \mathbf{v}\left(\omega_{0}\right)$ of the $2 \times 2$ matrix $1-\widetilde{\mathcal{S}}\left(i \omega_{0}\right) \mathbf{J}$. One finds that $K=0$, the nonzero elements of $\mathbf{L}$ are

$$
L_{1111}=2 \kappa^{3} W, \quad L_{2111}=-2 \kappa^{3} \hat{W}
$$

and $\mathbf{u}\left(\omega_{0}\right)=\left(\begin{array}{c}\left(i \omega_{0}+\varepsilon\right) / \gamma \\ 1\end{array}\right), \mathbf{v}\left(\omega_{0}\right)=\left[\begin{array}{c}1 \\ \left(i \omega_{0}-\varepsilon\right) / \gamma\end{array}\right]$.

Setting $\hat{W}=2 \varepsilon / \kappa$ and substituting Eqs. (5.15), (5.16), (5.20), and (5.21) into (5.6) yields

$$
\sigma=\frac{i \kappa^{2}}{\omega_{0}}\left[\omega_{0}^{2}+\gamma^{2}-\varepsilon^{2}-2 i \varepsilon \omega_{0}\right] .
$$

Hence, $\operatorname{Re}(\sigma)=2 \varepsilon \kappa^{2}>0$, and we conclude that the system undergoes a supercritical Hopf-bifurcation as $\hat{W}$ increases beyond the value $2 \varepsilon / \kappa$. (The other conditions required by the Hopf bifurcation theorem can be shown to hold.) We conclude that a compartmental model neuron (or a homogeneous network) with excitatory feedback to the soma and inhibitory feedback to the dendrites can undergo a Hopf bifurcation leading to the formation of a stable limit cycle.

Example (ii): In the previous example, oscillations arose as a consequence of the particular pattern of feedback across the dendrites and soma. Since there was only a single dendritic compartment, the resulting convolution kernels corresponded to the weak delay case, see Eq. (5.15). Thus, we required nonzero feedback to the soma $(\hat{W} \neq 0)$, otherwise oscillations could not occur via a Hopf bifurcation, (see Fig. 3). For our second example, we consider a multicompartmental model neuron with feedback to the dendrites but not to the soma, and use the Hopf bifurcation theorem to investigate whether or not oscillatory behavior is induced by the presence of strong delays. (This corresponds to the situation of marginal stability at the intersection point $W^{-}$of Fig. 3.) The dynamics of the model neuron is described by Eq. (2.16) with $\hat{W}=0$, and no external inputs $F(t)=0$. Again the $H$ term is neglected for convenience. The dendrites are represented as a uniform infinite chain of compartments as in the example at the end of Sec. IV with $\alpha_{0}=0$ and $w_{\beta}=\delta_{\beta, L}$ for some integer $L$ so that $G(t)=G_{0 L}^{(0)}(t)$. Equation (2.16) then has the equivalent form (5.4) with

$$
S(z)=\left(\begin{array}{cc}
0 & (z+\hat{\varepsilon})^{-1} \\
\gamma W \widetilde{G}(z) & 0
\end{array}\right), \quad \mathbf{J}=\left[\begin{array}{ll}
\kappa & 0 \\
0 & 1
\end{array}\right] .
$$

The characteristic function is $\Delta(z)=z+\hat{\varepsilon}-\kappa W \widetilde{G}(z)$, which was analyzed in Sec. IV. In particular, for $L \neq 0$ the presence of a strong delay kernel means that there exists a weight value $W^{-}$of the form (4.16) for which $\Delta\left( \pm i \omega_{0}\right)=0$, where $\omega_{0}$ is the smallest nonzero solution to Eq. (4.17). The condition for a supercritical Hopf bifurcation is $\operatorname{Re} \sigma>0$ where

$$
\sigma=-\frac{\kappa^{2} \widetilde{G}\left(i \omega_{0}\right)}{4 \widetilde{G}\left(i \omega_{0}\right)}
$$

Using the definitions of $C(\omega)$ and $S(\omega)$ in Eq. (4.11), we have that

$$
\widetilde{G}(i \omega)=C(\omega)-i S(\omega), \quad \widetilde{G}^{\prime}(i \omega)=-\frac{d S(\omega)}{d \omega}-i \frac{d C(\omega)}{d \omega}
$$

and the condition $\operatorname{Re} \sigma>0$ reduces to 


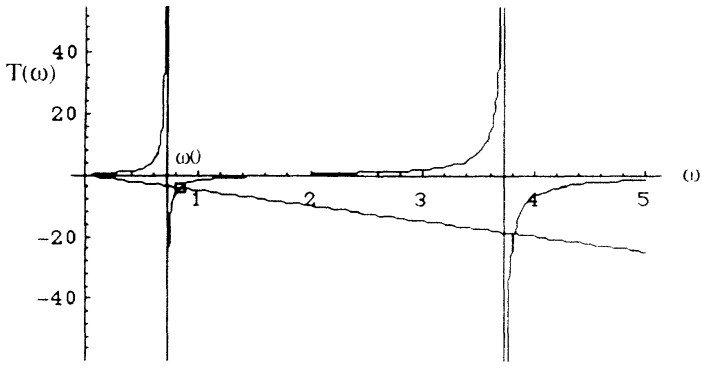

FIG. 8. Plot of the function $T(\omega)$ for a compartmental model neuron with feedback to the $L$ th dendritic compartment and delay kernel $G_{0 L}^{(0)}(t), L=4$. Graph shows that $d T / d \omega>0$ everywhere except at a finite set of singularities. The intercept $\omega_{0}$ of $T(\omega)$ with the straight line determines the pair of pure imaginary roots $\pm i \omega_{0}$ of the resulting characteristic equation at the point of marginal stability $W^{-}$.

$$
\frac{d T(\omega)}{d \omega}=\frac{C(\omega) S^{\prime}(\omega)-S(\omega) C^{\prime}(\omega)}{C^{2}(\omega)}>0 \text { at } \omega=\omega_{0} .
$$

By plotting $T(\omega)$ as a function of $\omega$ one finds that for any $L>0$, the smallest nonzero solution to Eq. (4.17) occurs at a point where $d T(\omega) / d \omega>0$ (e.g., see Fig. 8). This is also seen explicitly in the case of the generic strong delay kernel $\quad G(t)=T^{-2} t \exp (-t / T) \quad$ since $T(\omega)$ $=2 \omega T /\left(1-\omega^{2} T^{2}\right)$ and $d T(\omega) / d \omega>0$ for all $\omega \neq 1 / T$.

We conclude that the presence of strong delays arising from the passive membrane properties of a neuron's dendritic tree system can lead to the onset of oscillations in a neuron with inhibitory dendritic feedback and zero somatic feedback. An analogous result holds at the network level when the interneuron connection weights are symmetric. (In the network case, the feedback need not be purely inhibitory.) The point of marginal stability for oscillation onset will occur when the most negative (real) eigenvalue of the connection weight matrix (the bifurcation parameter) takes on the value $W^{-}$while the most positive eigenvalue is below the positive intercept $\boldsymbol{W}^{+}$. Note that this result is not inconsistent with the global analysis of Ref. [11], since the construction of a Liapunov functional for a symmetric network is carried out in Ref. [11] using a weak delay kernel.

\section{DISCUSSION}

In summary, we have developed an integral equation approach for analyzing the dynamics of recurrent compartmental model neural networks, which allows the effects of dendritic structure to be incorporated in a compact and analytically tractable manner. The resulting kernels are generated from the single neuron membrane potential response function, which may be calculated for arbitrary dendritic tree topologies. Using linear stability analysis and a version of the Hopf bifurcation theorem, we have shown that an equilibrium of a compartmental model network can destabilize leading to the onset of a stable limit cycle. In the case of weak delay kernels, this only occurs when the bifurcation parameter is a complex eigenvalue of the interneuron connection weight matrix
$\mathbf{W}$ (assuming that $\hat{\mathbf{W}}=\mathbf{0}$ ). On the other hand, a Hopf bifurcation can arise with respect to variation of a real eigenvalue (as necessarily occurs when the weight matrix is symmetric) in the presence of strong delay kernels. The stability region of an equilibrium is found to shrink as the effective delay $T$ increases reaching a minimum at some finite value of $T$. Thereafter, the stability region grows with $T$. This contrasts with the case of a discrete delay where the equilibrium becomes increasingly less stable as the delay increases [10].

There are a number of possible extensions of this work which include the following.

(i) To carry out a more detailed analysis of the dependence of compartmental model network dynamics on the pattern of interneuron connection weights and internal dendritic structure. (The latter includes both the topology of the dendritic tree and the various time constants of the system.) For example, the stability of particular network configurations can be explored along similar lines to the discrete delay case by calculating the eigenvalue spectrum of the weight matrix [10].

(ii) In this paper, we considered an analog model that takes the output of each neuron to be a sigmoidal function of the somatic membrane potential $U$. An alternative choice is to take an integrate-and-fire model in which the somatic membrane potential resets to some level $\bar{U}$ each time a neuron fires [18]. That is, $U\left(t^{+}\right)=\bar{U}$ whenever $U(t)=h$, where $h$ is some threshold. The output of each neuron is now in the form of a sequence of action potential spikes (idealized as Dirac $\delta$ functions), which is specified by the interspike time intervals. Recently, we have used integral equation techniques to analyze the dynamics of a single compartmental model integrate-andfire neuron and, in particular, to determine how reset feeds back on to the dendrites [19]. We are currently extending this analysis to the network level.

(iii) The study of the onset of oscillations in compartmental model neural networks is of particular interest within the context of collective synchronization. Inspired by certain neurophysiological experiments [20,21], the formation of coherent states in which spatially separated groups of oscillating neurons are phase synchronized has been suggested as a possible mechanism for feature linking in pattern recognition tasks. However, most analytical models of such processes neglect the internal dendritic structure of neurons, see for example Refs. [22-24]. The integral equation techniques presented here could be used to investigate synchronization in networks consisting of more realistic, compartmental model neurons.

(iv) In the compartmental model presented in Sec. II, we neglected the fact that changes in the membrane potential $V_{\alpha}$ of a compartment induced by a synaptic input depends on the size of the deviation of $V_{\alpha}$ from some fixed resting potential. If this so called shunting effect is included then there are additional nonlinear terms on the right-hand side of Eq. (2.2) of the form $V_{\alpha} f(U(t))$. Thus, Eq. (2.2) is no longer linear in the $V_{\alpha}$, which makes their elimination through integration much more complicated. We are currently looking at ways to tackle this problem. 
[1] W. Rall, in Neural Theory and Modelling, edited by R. F. Reiss (Stanford University Press, Stanford, 1964).

[2] C. Koch and I. Segev, Methods in Neural Modelling (MIT, Cambridge, MA, 1989).

[3] P. C. Bressloff and J. G. Taylor, Biol. Cybern. 69, 199 (1993).

[4] P. C. Bressloff, Network 4, 155 (1993).

[5] P. C. Bressloff, J. Phys. A 27, 4097 (1994).

[6] P. C. Bressloff and J. G. Taylor, Phys. Rev. E 47, 2899 (1993).

[7] T. Poggio and V. Torre, Lect. Notes Biomathematics 21, 89 (1977).

[8] J. K. Hale, Theory of Functional Differential Equations (Springer-Verlag, New York, 1977).

[9] J. M. Cushing, Lect. Notes Biomath. 20, 1 (1977).

[10] C. M. Marcus and R. M. Westervelt, Phys. Rev. A 39, 347 (1989).

[11] T. A. Burton, Neural Networks 6, 677 (1993).

[12] D. J. Allwright, Math. Proc. Cambridge Philos. Soc. 82, 453 (1977).

[13] M. A. Cohen and S. Grossberg. IEEE Trans. Syst. Man Cybern. 13, 815 (1983); J. J. Hopfield, Proc. Natl. Acad.
Sci. 79, 2554 (1984).

[14] L. F. Abbott, E. Farhi, and S. Gutmann, Biol. Cybern. 66, 61 (1991).

[15] T. A. Burton, SIAM Rev. 25, 343 (1983).

[16] R. K. Miller, in Delay and Functional Differential Equations and Their Applications, edited by $\mathrm{K}$. Schmitt (Academic, New York, 1972).

[17] R. K. Miller, J. Diff. Eqns. 15, 129 (1974).

[18] J. P. Keener, F. C. Hoppenstead, and J. Rinzel, Siam J. Appl. Math. 41, 503 (1981).

[19] P. C. Bressloff, LUT Department of Mathematical Sciences Report No. A210, 1994 (unpublished).

[20] C. M. Gray, P. Konig, A. K. Engel, and W. Singer, Nature 338, 334 (1989).

[21] R. Eckhorn, R. Bauer, W. Jordan, M. Brosch, W. Kruse, M. Munk, and R. J. Reitboeck, Biol. Cybern. 60, 121 (1988).

[22] P. Konig and T. B. Schillen, Neural Computation 3, 155 (1991); 3, 167 (1991).

[23] H. G. Schuster and P. Wagner, Biol. Cybern. 64, 77 (1990); 64, 83 (1990).

[24] A. Atiya and P. Baldi, Int. J. Neural Syst. 1, 103 (1989). 